\title{
Philosophiques
}

\section{L’utopie de la gynilité, un non-lieu provisoire}

\section{Louky Bersianik}

Volume 21, numéro 2, automne 1994

Les femmes et la société nouvelle

URI : https://id.erudit.org/iderudit/027287ar

DOI : https://doi.org/10.7202/027287ar

Aller au sommaire du numéro

Éditeur(s)

Société de philosophie du Québec

ISSN

0316-2923 (imprimé)

1492-1391 (numérique)

Découvrir la revue

Citer ce document

Bersianik, L. (1994). L’utopie de la gynilité, un non-lieu provisoire. Philosophiques, 21(2), 459-469. https://doi.org/10.7202/027287ar
Résumé de l'article

Constatant la perdurance du patriarcat, l'auteure souligne la pertinence actuelle du féminisme et la nécessité d'une utopie de la gynilité, dont elle indique le rapport à la littérature. d'utilisation que vous pouvez consulter en ligne.

https://apropos.erudit.org/fr/usagers/politique-dutilisation/ 


\title{
L'utopie de la gynilité, un non-lieu provisoire
}

\author{
par \\ Louky Bersianik
}

\begin{abstract}
RESUME : Constatant la perdurance du patriarcat, l'auteure souligne la pertinence actuelle du féminisme et la nécessité d'une utopie de la gynilité, dont elle indique le rapport à la littérature.
\end{abstract}

ABSTRACT : Patriarchy is still living and in good health. That is why feminism is still actual, and why a gyno-utopia is all the more necessary, both for society and for literature.

C'est difficile de parler à la suite de deux brillantes écrivaines sachant improviser. Bien que je n'aie pas le don de l'improvisation, je vais vous faire un peu de science-fiction à ma manière, à l'aide d'un extrait du Pique-nique sur I'Acropole (I979) ${ }^{\mathrm{I}}$, paru récemment en livre de poche. Ce passage, curieusement, préfigurait l'une des questions du bloc « Prospective » de la proposition de ce colloque, question formulée comme suit : «Quels devraient être les rôles des hommes dans la société nouvelle? » Voici donc cet extrait:

Quand toutes les femmes auront débarqué sur la terre, dit Aphélie, je parie qu'il y aura plus de remue-ménage que quand les ci-devant hommes ont débarqué sur la lune.

La question alors sera de savoir quelle deviendra la place des hommes dans un monde où il y aura aussi des femmes. Je pense que ce n'est pas aux femmes de répondre à cette question, dit Ancyl.

C'est vrai dit Aphélie. L'histoire de l'humanité qu'on nous a donnée pour uraic est un grand roman de science-fiction plein de monstres fabuleux et de beautés extraterrestres; un grand roman policier aussi, plein de meurtres anonymes où l'on a fait disparaître les corps, où l'on a parfaitement fait disparaître les taches de sang, de sorte que les gens ne croient pas à la réalité de ces meurtres.

Pour de tels meurtriers, dit Ancyl, leurs meurtres sont « symboliques 》 comme ils disent (PA, 77).

Oui, « symboliques », justement... Je vous le disais bien qu'on était en pleine science-fiction.

I. Louky Bersianik, Le Pique-nique sur l'Acropole, (VLB, 1979), Editions de l'Hexagone, coll. Typo, préface de Claudine Potvin, Montréal, 1992. La référence de la citation renvoie à la pagination de cette nouvelle édition. 
Comme convenu, cette contribution comportera plusieurs extraits de mes livres, surtout du dernier, La main tranchante du symbole (I990) ${ }^{2}$, qui réunit des textes et des essais féministes écrits durant les dix dernières années. La référence abrégée : «MTS », est presque un acronyme... et laisse croire qu'avec ce livre je transmets quelque chose qui finira par polluer le système patriarcal dans lequel nous vivons...

\section{Qu'est-ce que la gynilité ?}

C'est dans L'Euguélionne $(1976)^{3}$, je crois, à la toute fin, que j'ai esquissé l'emploi de ce mot gynilité pour la première fois : « Je suis gyr z et le mâle de mon espèce est andre, dit l'Euguélionne. [...] Et moi qui suis gynile, je viens vers lui qui est viril » (EUG, 388-389). Un néologisme dont j'ai développé le sens quelques années plus tard à I'endos du Calendrier I979 des Editions du Remueménage qui avait « la féminité » pour thème, cette année-là. Voici un extrait de ce texte, qui a été repris dans La main tranchante du symbole:

La « FÉminité 》, ce mot tendancieux let ambigu], voire péjoratif pour plusieurs [...], tout chargé qu'il soit de ses mystères vrais ou faux, ne fait pas le poids avec son homologue masculin, parce qu'il n'inclut pas du tout la puissance sexuelle des femmes, leur énergie, leur force, leur courage, leur vigueur, leur hardiesse, leur audace, leur noblesse, etc., comme le fait si abondamment le mot « viriIITÉ » pour les hommes.

Voilà pourquoi l...l il faut garder en mémoire la « GYNILITÉ » des femmes, qui est leur identité féminine reconnue, leur féminité observée de l'intérieur et non imposée de l'extérieur par les hommes, leur spécificité féminine et humaine sans référence à la masculinité (MTS, 249).

Ce concept, en apparence $u$-topique, semble encore aujourd'hui un projet irréalisable. Mais c'est un non-lieu provisoire. Comme je l'ai déjà dit lors d'un entretien ${ }^{4}$ : « Je suis certaine que, dans les temps les plus reculés, le patriarcat était de l'ordre de l'utopie dans l'imaginaire des hommes. »

Ils ont dû rêver d'abord qu'ils enfantaient, rêver pendant longtemps qu'ils étaient capables d'enfanter avant de s'approprier l'enfantement. Ils ont dû rêver très fort qu'ils étaient des dieux avant de soumettre les femmes à leur loi : d'abord des faux dieux, puis des vrais, puis un seul Dieu Tout-Puissant en plusieurs personnes mâles d'une très grande variété. Et quand on se frotte à leur littérature, on ne peut s'empêcher de penser qu'ils ont dû, très tôt, imaginer le monde comme un immense bordel dans lequel des créatures de rêve ou de

2. Louky Bersianik, La main tranchante du symbole, Editions du Remue-ménage, Montréal, rggo.

3. Louky Bersianik, L'Euguéliomme, La Presse 1976, Editions internationales Alain Stanké, coll. Québec ıo/ro, Montréal, 1985. La référence de la citation renvoie à la pagination de la première édition.

4. « Le tremblement de la conscience/Entretien avec Louky Bersianik », par Louise Dupré, dans Voix \& Images, [Dossierl Louky Bersianik, Editions de l'Université du Québec à Montréal, Automne ig9ı, n 49, p. 20. 
cauchemar, mises là tout exprès pour eux, étaient à leur entière disposition; que c'était même un avantage pour elles que d'être utilisées à fond et sans ménagement par eux. «Pour les rendre intelligentes, rien de tel que de les faire couvrir », confie à un autre un des personnages de Maupassant.

Selon le dictionnaire, l'utopie est une vue politique ou sociale qui ne tient pas compte de la réalité. Dans la mesure où notre réalité est happée puis niée par l'ensemble du système qui repose sur le code symbolique patriarcal, peuton sérieusement affirmer que l'utopie de la gynilité ne tient pas compte de la réalité ?

Le patriarcat avait ébranlé les assises de l'être féminin en lui retirant le sol sous les pieds, tandis qu'à l'être masculin il avait érigé un piédestal. L'utopie de la gynilitéa voulu rendre visible cette réalité, la sortir des décombres de la négation et lui substituer une autre réalité à travers sa propre mosaïque de transformation, son propre langage symbolique, déjà reconnaissable, sinon reconnu.

S'il est fait de part et d'autre du masculin et du féminin, ce travail de transformation et de reconnaissance arrivera peut-être à déjouer les ruses ancestrales du patriarcat auxquelles nous nous laissons encore prendre car elles nous collent au corps comme une seconde peau.

\section{Le patriarcat existe-t-il encore?}

Je soutiens qu'il existe plus fort que jamais. Si ses institutions commencent à faire un peu de place aux femmes dans les sphères de décision, c'est avec parcimonie et dans l'espoir d'assimiler les meilleures d'entre elles au pouvoir économique, politique et du savoir. Ce pouvoir, même partagé avec les femmes, est toujours patriarcal. Presque toutes les femmes ont accepté de signer un contrat qui ne profite qu'à un petit nombre d'entre elles, le contrat d'un patriarcat renouvelé pourrait-on dire.

Je cite un passage de l'Introduction de La Main tranchante du symbole:

Le patriarcat est une infrastructure antidémocratique qui sous-tend toutes les formes de société, que celle-ci soit capitaliste, socialiste, communiste, totalitaire, libertaire. La forme de société peut changer, l'infrastructure demeure, immuable, solide, infrangible. Il en sera toujours ainsi tant que l'on s'obstinera à ne pas pointer le patriarcat comme système à changer plutôt que les autres formes de société, nées de doctrines ou de théories sociales et économiques souvent divergentes, mais dont les révolutions ne font qu'échanger le rapport de forces sans vraiment en entamer le caractère patriarcal qui est mesquin, cruel et mortifère.

En tant que système économique et social sous-jacent, le patriarcat est une phallocratie extrêmement efficace et dangereuse, parce qu'elle accrédite symboliquement la loi du plus fort. La culture qu'elle impose à toutes les autres cultures est une injure à la vraie démocratie. Rien d'étonnant à ce que celle-ci soit si boiteuse et n'ait vraiment lieu nulle part en ce monde (MTS, I6. I7).

Le nouvel ordre mondial n'est pas pour demain. Celui qu'on appelle ainsi depuis la guerre du Golfe date déjà de plusieurs millénaires, du jour où Athéna 
a fait pencher la balance du côté des pères après avoir renié sa mère et nié le matricide.

\section{Le féminisme est-il devenu inutile?}

L'ontologie des femmes fait l'objet d'un «non-lieu », pour insuffisance de preuves pourrait-on dire. Elle est toujours instable, sujette à caution, souffrant de dédoublement. Elle reste problématique dans l'intellect des philosophes dès qu'ils songent que les femmes aussi sont des hommes, ou que les femmes sont aussi des hommes... Alors, ils renvoient cette sempiternelle ambiguité aux linguistes avec mission de réparer la langue des accidents du passé. Mais les linguistes, en mauvais mécaniciens, trouvent tout naturel que cette mécanique du langage soit boiteuse, ou plutôt, ils ne voient même pas qu'elle boite avec son pied bot qui contrefait l'humanité tandis que l'autre est bien formé et mâle par surcroît.

A propos de ces êtres qui vont clopin-clopant, voici une petite anecdote navrante à saveur ontologique. Lors d'une grande exposition collective à Montréal, une invitée demanda à l'organisateur comment il se faisait que, sur soixante exposants, il n'y avait que six femmes. Il lui fut répondu ceci : « Il n'y a pas de boiteux non plus! $»$.

Lysiane Gagnon, à La Presse, ne raisonnait pas autrement dans son éditorial du 8 septembre 1990 concernant la composition de la Commission BélangerCampeau sur l'avenir du Québec:

L'absence des femmes ? C'est un faux problème. On trouve chez les femmes la même diversité d'opinions, sur la question nationale, que chez les hommes, et il n'y a pas d'« angle féminin ». Si l'on avait ouvert la porte à toutes les «minorités », la confusion serait totale : pourquoi pas, alors, les vieux, les jeunes, les écolos, les chômeurs, les gais, les musulmans, les féministes radicales, les féministes modérées, etc., etc. ?

Ce n'est sans doute pas de la mauvaise foi. Peut-être est-ce le fait d'une infirmité intellectuelle... Une claudication de la cervelle ? Précisons qu'il s'agit certainement d'une cervelle « minoritaire ».

Que la femme soit considérée comme l'une des deux composantes de l'humanité, cela relève encore de l'utopie. En fait, elle n'en est qu'une variété parmi d'autres comme les handicapés, les autochtones, les minorités ethniques et culturelles, les jeunes, les gens du troisième âge, etc. Jamais autant n'y est-elle apparue telle, à travers les médias, que depuis le début de ces discussions interminables sur la Constitution canadienne.

Or, comme tous les autres peuples, et avant tout autre considération, le peuple québécois est composé d'abord de femmes et d'hommes. La répartition sexuelle est la première caractéristique, non seulement de l'humanité, mais de la plupart des êtres vivants. On chercherait en vain l'inscription de cette distinction primordiale dans la Charte québécoise des droits et libertés. 


\section{l'utopie de l'égalité}

$\mathrm{Si}$, malgré les chartes visant à la protection des droits de la personne, une société véhicule des préjugés envers les femmes et tolère que s'exercent de la discrimination et de l'injustice envers elles, et ce, jusque dans ses Cours de justice, c'est en partie parce que, dans ces chartes, les droits de cette moitié de la population sont noyés dans ceux des minorités comme si elle n'était qu'une minorité parmi d'autres et comme si ces autres minorités étaient exclusivement masculines. N'est-ce pas intolérable cette façon que l'on a de classer les femmes avec les minorités et sur le même pied que celles-ci quand ce n'est pas à un niveau inférieur?

Ainsi, à l'article io de notre Charte québécoise des droits et libertés, au chapitre du Droit a l'égalité où sont nommés sept motifs de discrimination, la mention du « sexe » vient après celles de « la race » et de « la couleur ». C'est une aberration philosophique puisque le sexe est une généralité qui précède la race et la couleur et que la discrimination raciale s'exerce autant sur l'un que sur l'autre sexe. De plus, c'est une insulte aux femmes, car on sait bien que la discrimination sexuelle se fait généralement à sens unique.

A noter que « la grossesse » vient en quatrième et que, si l'on se réfère à la clause $n^{\circ}$ I du même article, il est dit que «nul ne doit harceler une personne en raison de l'un des motifs visés dans l'article ior ». Comment se fait-il que l'on n'évoque jamais cette clause lorsque les femmes enceintes qui veulent se faire avorter se font harceler par les «pro-vie »?

\section{La coupure épistémologique}

A mon sens, l'utopie de la gynilité sera en bonne voie de réalisation le jour où s'opérera une vraie coupure épistémologique dans le champ entier du savoir et non plus uniquement dans les marges de ses institutions, où se situent les chaires d'étude sur la condition des femmes et les Women Studies. Et le jour où ce savoir élargi traversera cette planète scintillante qui nous habite et que nous appelons la conscience humaine. La conscience est le cœur battant de notre univers et elle ne se nourrit pas seulement des fruits de la connaissance.

Comme je l'écrivais à Maria De Koninck en réponse à son invitation, s'il ne se produit pas, dans le champ théorique, une rupture épistémologique plus radicale, si l'on ne reconnaît pas que nos démocraties occidentales actuelles sont avant tout patriarcales et le demeurent malgré les acquis du féminisme, et si l'on continue de bâtir le futur sur cette situation fausse en en reproduisant toujours les mêmes erreurs, il n'y aura pas de société meilleure ni de réalisation concrète de lutopie de la gynilité.

"Nous avons, que nous le voulions ou non, une responsabilité historique », nous dit Christine Delphy dans Chroniques d'une imposture (1981 $)^{5}$.

5. Christine Delphy, «Libération des femmes An dix », dans Chroniques d'unc imposture. Du Mouvement de libération des femmes à une marque commerciale, Edité par l'Association Mouvement pour les Luttes Féministes, Paris, 198I. Texte d'abord publié dans Questions féministes, n 7, fév. 1980. 
Elle ajoute : «Car c'est à nous, féministes d'aujourd'hui, que les femmes de demain seront en droit de demander des comptes; si nous laissons une deuxième fois en cent ans le féminisme s'enliser et disparaître dans les sables, si, ayant nous-mêmes profité - et pâti - de l'histoire et de son maniement, nous ne faisons pas tout ce qui est en notre pouvoir pour qu'elles n'aient pas, en 2030 ou en 30I7, à repartir à nouveau à zéro. »

\section{l'utopie de la fiction}

En tant qu'écrivaine, je sens bien que c'est utopique de penser que mon écriture aille de soi et s'inscrive avec d'autres sur le grand monument de la littérature.

Pourquoi est-ce si difficile pour une écrivaine féministe d'écrire de la fiction, est-ce même impossible pour elle que sa fiction « rime », c'est-à-dire corresponde, réponde à un consensus général comme la langue, la culture, la tradition qui sont des conditions de création; une fiction qui s'accorde, soit conforme, s'harmonise avec le code symbolique qui est lui-même une feinte, une fiction, une effigie, une image en relief et exclusive d'une seule des deux composantes de l'humanité?

Dans cette perspective, qu'est-ce que le «second degré » pour une écrivaine féministe? Pourquoi la théorie-fiction qui mélange les genres estelle une pratique exclusivement féminine ou presque? A propos de « second degré », voici deux autres extraits de La main tranchante du symbole:

Le symbole sépare et rassemble comme la main qui est « le côté tranchant du cerveau humain », selon le mot de Bronovski. Et qui en est aussi son chant puisque, de son exercice raffiné, naissent la plupart des formes d'art.

Ainsi en est-il du symbole dont l'une des principales fonctions réside dans son dynamisme transformateur. Transformer les ténèbres en lumière, «énergiser » d'un nouveau flux la fonction symbolisante de l'imagination, infléchir les symboles existants, les manier suivant une nouvelle logique symbolique de façon à ce qu'ils émergent avec de nouvelles résonances, de nouvelles interprétations, telle est la tâche des créatrices féministes (MTS, 216).

[Maisl il faut bien admettre qu'une autre des principales fonctions du symbole est de créer un deuxième degré là où il y a un manque de réalité, et de hausser ce manque au-dessus de la réalité en en faisant une loi. Le Nom-dupère en est justement un exemple éloquent : tandis que la mère est réelle, le père est fictif et c'est lui qui fait la loi aux mères, sous prétexte qu'il doit faire la loi aux enfants. Fictif puisqu'il procède d'une feinte, d'une hypothèse, d'une imitation de la matrice et d'une superposition à la mère (MTS, 174).

Ce passage trouve son écho en quatrième de couverture dans un autre extrait de ce livre:

Contrairement à la paternité, la maternité n'a jamais fait l'objet d'une découverte historique, d'une révélation lj'emploie ici le mot révélation dans son sens religieux]. Mais le procès d'Oreste a jeté les bases d'une captation symbolique du lien maternel au profit de l'homme, ce qui n'a pu et ne peut encore se faire sans une violence inouie. 
Fille ou garçon, l'enfant est regardé comme un dérivé du père. Le nom qu'on lui donne est sa première dérive, son premier détournement. Nayant pas pris racine en l'homme, l'enfant se trouve annexé à lui dès sa sortie du ventre féminin, par un nouveau cordon ombilical qui ne sera jamais coupé et qui lui fera reproduire tout au long de son existence les formes du paradigme paternel.

Dans sa tentative de rouvrir le procès d'Oreste, ce livre voudrait jeter un nouvel éclairage sur les symboles issus des conclusions de ce procès, symboles qui ont façonné le patriarcat tel qu'il se présente aujourd'hui.

Le scénario de cette fiction initiale, principal obstacle à la réalisation de l'utopie de la gynilité, pourrait se dérouler comme suit sous le titre:

LE MEURTRIER AUX MAINS BLANCHES ET LE TRAVESTI

A pollon ordonne le meurtre de la mère et fait exécuter ce meurtre par le nourrisson devenu grand. Plus tard, devant le tribunal, il fait passer son crime pour une fable, une histoire inventee.

A théna dans son homélie dominicale trahit celles qui ont tous les siècles d'humanité à mettre au monde. Elle vend un nouveau produit injurieux pour elles, qu'elle appelle « la loi paternelle ».

A pollon fait son plaidoyer devant le tribunal de la nouvelle justice. Il récite avec arrogance sa nouvelle fable qui deviendra le texte fondateur du patriarcat :

Le matricide est impossible puisque la mère est étrangère à son propre enfant.

A théna dans son discours sur le nouvel ordre mondial renie sa mère et se fait enfanter par son père.

A pollon qui a la passion des fables présente sa nouvelle fable comme étant la réalité. Dans son plaidoyer pour l'imposture, il exécute la mère du nourrisson une seconde fois :

Seul le père a un lien de parenté avec son enfant

La mère n'est qu'une mère porteuse

A théna est un travesti mâle qui fait la femme pour rassurer les mères et pour endormir les récalcitrantes.

A pollon est un agréable boucher vêtu de blanc. Il n'a aucune trace de sang sur la figure. Il n'a aucune trace de sang sur les mains. Sur ses vêtements, il n'y a aucune tache de sang. Il est blanc comme neige. Il a les quatre pieds

blancs. Comme la bonne conscience.

Elle joue divinement du bouclier.

Il joue divinement de la lyre.

\section{l'utopie de l'esthétique sans l'éthique}

Que vaut l'esthétique sans l'éthique ? À quoi « rime » mon esthétique personnelle et qu'a-t-elle à voir avec l'émotion esthétique masculine qui trouve confirmation de la supériorité des hommes sur les femmes à travers l'art et la littérature?

En quoi les critères esthétiques généralement admis devraient-ils faire l'objet d'une sérieuse mise en question, dans la mesure où ils participent d'une fiction d'où les femmes sont exclues? 
Je sais que beaucoup de gens s'interrogent aujourd'hui là-dessus, surtout des philosophes. Mais qu'en feront-ils, qu'en feront-elles ? Peut-on leur faire confiance quand on sait que cette question est rarement abordée sous son « angle féminin », c'est-à-dire là où l'éthique souffre le plus en regard d'une soi-disant esthétique?

\section{L'utopie de l'universel et celle de la condition humaine}

Pourquoi l'écriture au féminin est-elle figée en dehors de l'« universel », n'est-elle pas homologuée par la culture, même quand cette écriture aborde les sujets métaphysiques ? Pourquoi les hommes en généra: et les critiques en particulier se sentent-ils étrangers devant une écriture qui traduit l'émergence d'une culture au féminin ? De quelle norme parlent-ils quand ils disent de cette écriture qu'elle est «à côté de la plaque »?

Et comment expliquer que la misogynie, «bien qu'elle ne soit pas une composante de la nature humaine, soit devenue un aspect inévitable et incontournable de la condition humaine de toutes les époques et sous toutes les latitudes » (MTS, 192). Je cite encore La main tranchante du symbole:

Si la littérature moderne et les développements de la linguistique let bien entendu de la psychanalysel ont obligé la critique à tenir un nouveau langage sur le langage, on ne peut pas dire qu'elle ait trouvé celui qui prendrait en charge les apports du féminisme à ce discours et en rendrait compte sans trop divaguer.

Or, ce discours est en connection directe avec l'imaginaire des femmes et, de ce fait, participe avec celui-ci de la dimension universelle, celle-là même qu'on accorde comme allant de soi à l'imaginaire des hommes, quand, par exemple, leurs poèmes sont nationalistes ou expriment leur révolte contre l'oppression... C'est cette dimension universelle, cet autre versant du langage, qu'on a tendance à nier aux œuvres des femmes [...] (MTS, 217).

Mais qu'est-ce encore que l'universel ? Il y a une quinzaine d'années, je suis allée voir pour la première fois une pièce que j'aime beaucoup et que j'ai revue plusieurs fois par la suite dans des mises en scène différentes. Il s'agit de Enattendant Godot, où l'on voit deux couples d'hommes qui vivent côte à côte depuis une cinquantaine d'années, bien que n'étant pas homosexuels.

Après la représentation, j'ai fait la remarque suivante à l'un des comédiens : «Pour un chef d'œuvre universel, je trouve que ça manque pas mal de femmes...« Il m'a répondu tout bonnement : « C'est parce que Beckett a voulu écrire une pièce sur la condition humaine, pas sur la sexualité... » J'ai eu l'impression qu'on me rejouait le coup de théâtre d'Adam et Ėve.

Cette question de la condition humaine pourrait se poser autrement, comme je l'ai entendu dans un film québécois : « Tout le monde, on est tout seuls devant la mort. Mais nous, les femmes, en plus, on est toutes seules devant la vie, qu'on la donne ou pas. » 


\section{Le directeur des ténèbres}

A la toute fin du long générique de la production des Atrides par le Théâtre du Soleil, après l'Intendance, le Contrôle et l'Entretien, il est fait mention du Directeur des Ténébres!

Diriger les ténèbres ! Quel métier ! Les rassembler, octroyer à chacune d'elles un lieu où l'on verra s'infiltrer les franges de la lumière, quel bonheur !

Mais aussi, dompter cette meute saisie d'effroi à l'approche de sa proie, tenir en respect les ombres froides qui se profilent dans l'avenir lointain dévolu à la nuit et les diriger vers la première aurore à l'horizon d'une mer encore en latence, telle est la tâche exaltante et difficile de l'archéologue du futur.

\section{L'archéologue du futur}

C'est dans la tête qu'on les entend, parfois, les cigales monocordes du patriarcat. Le son aigu de l'épée flamboyante d'Achille qui vous tue sans vous toucher, parce qu'elle est suspendue au-dessus de vos têtes, parce qu'elle insiste à se tenir sans cesse à vos côtés, parce qu'elle fait de l'ombre à votre ombre et couche entre vous et ce que vous aimez, ce qui vous rend malade d'amour; et parce que c'est pour cette maladie d'amour que l'on vous soigne au fond de la terre, aveuglément, au centre des ténèbres.

On ne peut pas refaire l'histoire, mais on ne peut s'empêcher de se demander où en serait le monde s'il avait été fait aussi par les femmes. Et de l'imaginer, dès les débuts, en train d'évoluer aux mains des unes et des uns, peut contribuer à réaliser l'utopie moderne de la gynilité alliée à l'utopie d'une virilité qui ne serait ni parade ni comédie burlesque, dans un monde qui se serait déplacé de quelques millimètres, selon le vœu de L'Euguélionne.

Essayer de construire un monde dans le futur, c'est l'arracher aux strates minérales et souterraines de l'invisible. C'est le construire dans les ténèbres des temps à venir comme l'archéologue du présent édifie une histoire nouvelle en extirpant des trésors anciens de la nuit des temps révolus.

Réaliser cette utopie dans la mémoire du futur, c'est aussi rendre visible l'imaginaire spécifique des femmes. Imaginer une mémoire du futur, c'est avoir en mémoire un avenir qui ne serait pas entièrement secrété par le passé, une partie de celui-ci n'étant pas encore advenu, c'est mémoriser le futur, le vingtet-unième siècle par exemple, et s'en servir pour penser et agir dans le présent. C'est une vision optimiste des choses car elle suppose que les femmes s'appartiendront totalement, non seulement physiquement et dans toutes les contrées de la terre mais symboliquement et à travers tous les langages.

C'est cette vision qui m'aide à vivre et c'est dans cette vision que je trouve mon inspiration pour écrire ce que j'appelle l'archéologie du futur. Quand j'emploie cette expression, je me réfère à un texte paru pour la première fois en I984 dans la Nouvelle Barre du Jour et qui a été reproduit dans La main tranchante du symbole: 
Je travaille sur la feinte et l'ambiguïté des choses actuelles qui deviendront vétustes, pour que naisse une science fictive des monuments antiques de l'avenir.

Jécris pour une archéologic du futur, pour que la mémoire du futur s'inscrive dans le présent de façon à ce que ce présent devienne une chose ancienne et dépassée.

Rendre visible la coïncidence des termes ambigus de la racine du mot archéologie : le commencement ll'ancienl et le commandement. Comme l'archaïque et le hiérarchique. Comme deux topiques mortifères de la même séquence patriarcale.

Rompre cette séquence et glisser dans la fissure le commencement lle nouveaul d'un monde viable. Permettre aux créatures marmoréennes de Phidias de se couvrir de chair et de sang. Transformer le motus du marbre en mot et cette statue en sujet femme.

Géante, terriblement vivante (MTS, 25).

Mon travail actuel d'écriture se situe justement dans le cycle d'une Archélogie du futur et s'accorde tout à fait avec l'idée que je me fais d'une épopée moderne (étymologiquement : émission de voix), c'est-à-dire d'un texte qui tient à la fois du poème et du récit, « où le merveilleux se mêle au vrai, la légende à l'histoire et dont le but est de célébrer un héros ou un grand fait » (P. Robert). En l'occurrence, texte où le « merveilleux » a sa raison d'être puisque c'est l'histoire de transformations radicales successives qui ont lieu comme par magie mais dont le mage a médité ses tours pendant des siècles avant de les donner en spectacle.

Ici, le « grand fait » que je tente de célébrer est historique et concerne plus de la moitié de l'humanité. Il s'agit de l'entrée en existence des femmes, non seulement comme groupe humain mais comme sujets singuliers. J'ai choisi de transposer ce fait poétiquement par le biais d'une double métaphore culturelle empruntée à la statuaire et à la mythologie grecques. Dans l'« épopée » présente, ces femmes de pierre et de légende seront l'objet d'une écriture nouvelle sur la surface des stèles qu'elles auront délaissées, redonnant à celles-ci une plus large place à l'écrit et une nouvelle fonction de mémoire. En d'autres termes pour que l'écrit chasse l'image monolithique et figée, ou purement instinctive et animale, qui a été transmise des femmes dans la culture.

Je n'ai pas le temps de vous lire quelques extraits de cette Archéologie $d u$ futur comme je voulais le faire, mais je terminerai mon intervention par la lecture d'un autre extrait, d'un poème cette fois, paru récemment dans l'anthologie de la revue Estuaire. Quand j'étais petite, on m'appelait Le p'tit Nan parce que j'ai eu une période du Non assez prononcée : « Nan Nan Nan Nan Nan »! L'extrait que je vais vous lire a été écrit en souvenir de la rue Delanaudière, à Montréal, où j'ai passé mon enfance, entre les rues Rachel et Marianne. Il exprime bien cette attitude de refus. 
Le poème s'intitule:

\section{I'ANNEAU D'HIER}

[...]

Je marche à l'infini entre le premier homme de ma vie et ma première femme et dernière vers l'obstacle majeur à faire sauter avec mes dix saisons d'âge aux poignets

Moi quand je joue c'est à petits sauts dans ma rue de semaine entre une héroine biblique et la République française deux belles plantes nommées Rachel et Marianne poussant leurs fleurs jaunes de macadam en aval et en amont de ma rue qui n'est pas un jardin

Moi c'est à petits sauts que je saute sur les cailloux d'un ruisseau de ville qui s'appelle l'enfance en songeant qu'hier assise parmi les enfants entre le jeu du furet et le jeu du refuge j'ai été choisie

L'anneau glissé dans ma paume par une pieuse grand-mère personne n'a deviné pourquoi j'ai joué toute seule au je du refus quand j'ai laissé choir sur les passants l'alliance dont elle avait cru sceller mes doigts de pluie 